\title{
Stochastic resonance in a model neuron with reset
}

\author{
H. E. Plesser ${ }^{1,2}$ and S. Tanaka ${ }^{3}$ \\ Laboratory for Neural Modeling, Frontier Research Program, RIKEN, \\ 2-1 Hirosawa, Wako-shi, Saitama 351-01, Japan
}

\begin{abstract}
The response of a noisy integrate-and-fire neuron with reset to periodic input is investigated. We numerically obtain the first-passage-time density of the pertaining Ornstein-Uhlenbeck process and show how the power spectral density of the resulting spike train can be determined via Fourier transform. The neuron's output clearly exhibits stochastic resonance.
\end{abstract}

Key words: integrate-and-fire neuron, Ornstein-Uhlenbeck process, stochastic resonance, renewal process

PACS: 87.10.+e, 05.40.+j

\section{Introduction}

Neurons are inherently stochastic information processing devices, whence the study of the influence of noise on neuronal signal transmission and computation is of great interest. Since the first evidence for the enhancement of signals by noise was presented about 15 years ago [1], the phenomenon of stochastic resonance has been demonstrated in a number of physical $[2,3]$ and biological systems, especially in sensory neurons [4-6]. In the wake of these experiments, the theory of stochastic resonance for dynamic systems has been well developed $[7,8]$, and was recently extended to aperiodic signals [9].

As neurons in higher centers of the brain need to maintain a high signal-tonoise ratio as well as peripheral ones, it is plausible to presume that stochastic

1 Corresponding author

2 E-mail: plesser@prairie.riken.go.jp

3 E-mail: shigeru@postman.riken.go.jp 
resonance is a general principle of biological information processing. Indeed, models describing neurons as bistable elements have been discussed in detail $[10,11]$. For quantitative comparison with neurophysiological data, though, model neurons closer to biological reality need to be investigated. To this end, we study in this letter the response to a sinusoidal stimulus with superimposed white noise of a widely used model neuron, the leaky integrate-and-fire neuron which is reset upon firing [12]. In this model, the development over time of the membrane potential is given by the solution of the Fokker-Planck equation describing the overdamped limit of the Ornstein-Uhlenbeck process with an absorbing boundary.

Unfortunately, no analytic solution to this boundary value problem is known [13], while existing approximate solutions are limited to particular parameter ranges; in particular, they require sufficiently strong input noise [14]. Therefore, we numerically solve for the first-passage-time density (FPTD), i.e. the mathematical equivalent of the inter-spike-interval distribution (ISI), using a computationally efficient integral equation approach. From the FPTD, we then calculate the power spectral density (PSD) of the spike train generated by the model neuron via fast Fourier transform, employing results from the theory of point processes. Finally, we determine the signal-to-noise ratio (SNR) of the neuron's output, which clearly exhibits stochastic resonance, i.e. SNR is maximal for a finite strength of input noise.

Note the crucial difference between the model studied here and the threshold detector model that has been studied by several authors in recent years [1517]. The former is reset after each firing, whence individual threshold crossings are uncorrelated and the entire spike train constitutes a renewal process. The latter, to which we shall refer as continuous-mode model, does not include a reset mechanism, but assigns one spike to each threshold crossing in positive direction. Thus, individual crossings are correlated and the membrane voltage is governed by the same Fokker-Planck equation as our model, but with natural boundaries at $\pm \infty$, permitting analytical treatment. Indeed, Jung [18] has given a theory of stochastic resonance in continuous-mode threshold detectors based on the periodic asymptotic solution of this Fokker-Planck problem.

\section{The model}

The membrane voltage $x(t)$ of the model neuron is governed by the Langevin equation of the overdamped Ornstein-Uhlenbeck process $[12,19,20]$

$$
\tau_{m} \dot{x}(t)=-x(t)+\mu+q \cos (\omega t+\varphi)+\xi(t),
$$


where we have set the resting potential to $x=0$. The membrane time-constant $\tau_{m}$ and the drift term $\mu$ are positive constants, while $q, \omega$ and $\varphi$ are arbitrary real constants, and $\xi(t)$ is Gaussian white noise with zero mean and autocorrelation $\left\langle\xi(t) \xi\left(t^{\prime}\right)\right\rangle=2 D \delta\left(t-t^{\prime}\right)$. The initial condition is $x(0)=0$, and the neuron fires upon reaching the threshold voltage $x(t)=x_{\mathrm{th}}$ : both $x$ and the phase of the input stimulus $\omega t+\varphi$ are reset to their values at $t=0$.

To normalize variable values, we scale time as $\bar{t}=t / \tau_{m}$ and voltage as $\bar{x}(\bar{t})=$ $x(t) / x_{\mathrm{th}}$, so that time constant and threshold become 1 , whence $\bar{\mu}=\mu / x_{\mathrm{th}}$, $\bar{q}=q / x_{\mathrm{th}}, \bar{\omega}=\tau_{m} \omega, \bar{\varphi}=\varphi$ and $\bar{D}=D \tau_{m} / x_{\mathrm{th}}^{2}$. Thus we obtain the the dimensionless equation

$$
\dot{x}(t)=-x(t)+\mu+q \cos (\omega t+\varphi)+\xi(t),
$$

where we have dropped the bars immediately for compactness of notation.

As mentioned above, each approach to the threshold is independent of the past, because of the reset upon firing. Therefore, assuming a spike train of infinite duration, the firing process is a stationary renewal process [21]. We

will solve the FPT problem in the next section before examining the spike train as a whole in sections 4 and 5 .

\section{First-Passage-Time Density}

In this section, we present an efficient numerical method for the computation of the FPTD

$$
\rho(t) \mathrm{d} t=\operatorname{Pr}\left\{x(t)=x_{\mathrm{th}}=1 \text { in }[t, t+\mathrm{d} t) \text { if } x(t=0)=0\right\},
$$

the theoretical counterpart of the ISI distribution.

The Fokker-Planck equation corresponding to the Langevin equation (2) is [22]

$$
\begin{aligned}
\frac{\partial}{\partial t} \mathcal{P}\left(x, t \mid x_{0}, t_{0}\right)= & -\frac{\partial}{\partial x}(-x+\mu+q \cos (\omega t+\varphi)) \mathcal{P}\left(x, t \mid x_{0}, t_{0}\right) \\
& +D \frac{\partial^{2}}{\partial x^{2}} \mathcal{P}\left(x, t \mid x_{0}, t_{0}\right)
\end{aligned}
$$

where $\mathcal{P}\left(x, t \mid x_{0}, t_{0}\right)$ is the probability density that the voltage is $x$ at time $t$ if it was $x_{0}$ at time $t_{0}<t$. The model is thus specified by the initial and boundary conditions $\mathcal{P}_{r}(x, t \mid 0,0)=\delta(x), \mathcal{P}_{r}(-\infty, t \mid 0,0)=0$ and $\mathcal{P}_{r}(1, t \mid 0,0)=0$, where the index $r$ indicates restriction to $x \in(-\infty, 1]$. No analytic solution is known for this boundary value problem and an approximation based on the method of images is valid for a limited range of parameters only [14]. 
Following Schrödinger [23], we thus construct an integral equation equivalent to the above boundary value problem, utilizing the solution $\mathcal{P}_{f}\left(x, t \mid x_{0}, t_{0}\right)$ of (4) for the unrestricted Ornstein-Uhlenbeck process on the entire real axis, i.e. with boundary conditions $\mathcal{P}_{f}\left( \pm \infty, t \mid x_{0}, t_{0}\right)=0$. The solution is [8]

$$
\mathcal{P}_{f}\left(x, t \mid x_{0}, t_{0}\right)=\frac{1}{\sqrt{2 \pi \sigma^{2}(t)}} \exp \left[-\frac{(x-\langle x(t)\rangle)^{2}}{2 \sigma^{2}(t)}\right],
$$

where the mean and variance of $x(t)$ are (writing $\eta=\cot ^{-1} \omega$ )

$$
\begin{aligned}
\langle x(t)\rangle= & \mu+\frac{q}{\sqrt{1+\omega^{2}}} \sin (\omega t+\varphi+\eta) \\
& +\mathrm{e}^{-\left(t-t_{0}\right)}\left[x_{0}-\mu-\frac{q}{\sqrt{1+\omega^{2}}} \sin \left(\omega t_{0}+\varphi+\eta\right)\right] \\
\sigma^{2}(t)= & D\left(1-\mathrm{e}^{-2\left(t-t_{0}\right)}\right) .
\end{aligned}
$$

Then, the FPTD $\rho(t)$ is given by the Volterra integral equation [22]

$$
\mathcal{P}_{f}(1, t \mid 0,0)=\int_{0}^{t} \mathrm{~d} s \mathcal{P}_{f}(1, t \mid 1, s) \rho(s)
$$

Due to the sine terms in $(6)$, the kernel $\mathcal{P}_{f}(1, t \mid 1, s)$ of the above equation cannot be rewritten as a function of $t-s$ alone and a solution by Laplace transform is not possible. A description of the FPTD via its moments cannot be obtained either, since such methods are based on the Laplace transform of the kernel $[24,25]$.

We thus solve for $\rho(t)$ using standard computational techniques. Since the kernel has an integrable square-root singularity at $t=s$, we rewrite (8) as

$$
\mathcal{P}_{f}(1, t \mid 0,0)=r(t) \rho(t)+\int_{0}^{t} \mathrm{~d} s \mathcal{P}_{f}(1, t \mid 1, s)[\rho(s)-\rho(t)]
$$

with $r(t)=\int_{0}^{t} \mathcal{P}_{f}(1, t \mid 1, s) \mathrm{d} s$. This integral can be evaluated numerically and, discretizing time as $t_{j}=j h$ with stepsize $h>0$, we obtain the following algorithm for calculating the FPTD [26]

$$
\rho_{0}=0, \quad \rho_{m}=\frac{h \sum_{j=1}^{m-1} K_{m, j} \rho_{j}-g_{m}}{\frac{h}{2} K_{m, 0}+h \sum_{j=1}^{m-1} K_{m, j}-r_{m}}, \quad m=1,2, \ldots
$$

where $K_{m, j}=\mathcal{P}_{f}(1, m h \mid 1, j h), g_{m}=\mathcal{P}_{f}(1, m h \mid 0,0), r_{m}=r(m h) . \rho_{0}=\rho(0)$ follows from the initial conditions.

The algorithm defined by (10) has proven to be stable and reliable. Over a wide range of parameter values, the calculated FPTDs $\rho_{m}$ are strictly nonnegative (if numerical noise of the order of machine accuracy is excluded) 
and the norm of the distributions approaches 1 from below as the range of calculation is extended towards larger $t$.

We found a different integral-equation approach $[27,28]$ to be slightly less stable for some interesting parameter values. In regions where both algorithms are stable, results agree well.

\section{Power Spectral Density}

To calculate the power spectral density (PSD) of the neuron's output, let us first consider a train of $M \delta$-spikes with inter-spike-intervals $\tau_{j}$ distributed according to the FPTD $\rho\left(\tau_{j}\right)$ :

$$
f_{M}(t)=\sum_{m=1}^{M} \delta\left(t-t_{m}\right), \quad t_{m}=\sum_{j=1}^{m} \tau_{j}, \quad t_{1}=0
$$

Neglecting the exact shape of the spikes amounts merely to dropping a form factor from the spectrum, while all statistically relevant information is contained in the firing times $t_{m}$ (see also [18]).

For $M \rightarrow \infty$, this process is a stationary renewal process, the spectra of which have been extensively discussed in mathematical literature [29,21], where they are known as Bartlett spectra [30]. Applications to neuronal systems have been rare to our knowledge [31].

The one-sided power spectral density of the spike train $f_{M}(t)$ is given by

$$
S_{M}(\Omega)=\overline{\tilde{f}_{M}(-\Omega)} \tilde{f}_{M}(-\Omega)+\overline{\tilde{f}_{M}(\Omega)} \tilde{f}_{M}(\Omega)=2 \overline{\tilde{f}_{M}(\Omega)} \tilde{f}_{M}(\Omega)
$$

where the bar indicates complex conjugation and

$$
\tilde{f}_{M}(\Omega)=\frac{1}{\sqrt{2 \pi t_{M}}} \int_{0}^{t_{M}} \mathrm{~d} t f_{M}(t) \mathrm{e}^{-i \Omega t}
$$

is the Fourier transform. Inserting (11) and (13) into (12) yields [21]

$$
\begin{aligned}
S_{M}(\Omega) & =\frac{1}{\pi t_{M}} \sum_{m, k=1}^{M} \mathrm{e}^{-i \Omega\left(t_{m}-t_{k}\right)} \\
& =\frac{1}{\pi} \frac{M}{t_{M}}\left\{1+\int_{0}^{\infty} \mathrm{d} t h_{M}(t) \mathrm{e}^{-i \Omega t}+\int_{0}^{\infty} \mathrm{d} t h_{M}(t) \mathrm{e}^{i \Omega t}\right\}
\end{aligned}
$$


where we have defined

$$
h_{M}(t)=\frac{1}{M} \sum_{j, k=1}^{M-1} \delta\left(t_{j+k}-t_{j}-t\right) .
$$

Integrating $h_{M}(t)$ over non-overlapping intervals would give the autocorrelation histogram of the neuron firing times. In the limit of an infinite spike train, we obtain

$$
h_{M}(t) \rightarrow h(t) \quad \text { and } \quad t_{M} / M \rightarrow\langle\tau\rangle \quad(M \rightarrow \infty),
$$

where $h(t)$ is the renewal density and $\langle\tau\rangle$ the mean first-passage-time. Note that the renewal density $h(t)$ is not a probability density, but $h(t) \mathrm{d} t$ is the probability for a spike to occur in $[t, t+\mathrm{d} t)$.

From the theory of renewal processes [21] we have for $\Omega \neq 0$

$$
\int_{0}^{\infty} \mathrm{d} t h(t) \mathrm{e}^{i \Omega t}=\frac{\tilde{\rho}(\Omega)}{1-\tilde{\rho}(\Omega)} .
$$

Here, $\tilde{\rho}(\Omega)$ is the Fourier transform of the FPTD $\rho(\tau)$. Performing the limit in (14) and inserting (17), we obtain the one-sided PSD of the infinite spike train

$$
S(\Omega)=\frac{1}{\pi\langle\tau\rangle}\left\{1+\frac{\tilde{\rho}(\Omega)}{1-\tilde{\rho}(\Omega)}+\frac{\tilde{\rho}(-\Omega)}{1-\tilde{\rho}(-\Omega)}\right\} \quad(\Omega>0) .
$$

Using this result, we can compute the PSD directly from the FPTD by means of a discrete Fourier transform.

For white shot noise, i.e. the Poisson process with FPTD $\rho(\tau)=\lambda \exp (-\lambda \tau)$, the terms in $\tilde{\rho}(\Omega)$ in (18) cancel and a white spectrum $S_{P}=1 / \pi\langle\tau\rangle$ results. Any deviation of $S(\Omega)$ from $S_{P}$ indicates the presence of a signal. For the Ornstein-Uhlenbeck process studied here, the spectra approach $S_{P}$ quickly for large $\Omega$ (Fig. 1). We will therefore employ $S_{P}$ as the reference noise level in section 5 .

\section{Stochastic resonance}

Having set the mathematical stage, we may now explore the response of our model neuron to sinusoidal input. A single parameter characterizing the input signal is the distance-from-threshold of the deterministic trajectory $\langle x(t)\rangle$

$$
\epsilon=1-\sup _{t \geq 0}\langle x(t)\rangle=1-\left(\mu+\frac{q}{\sqrt{1+\omega^{2}}}\right) .
$$




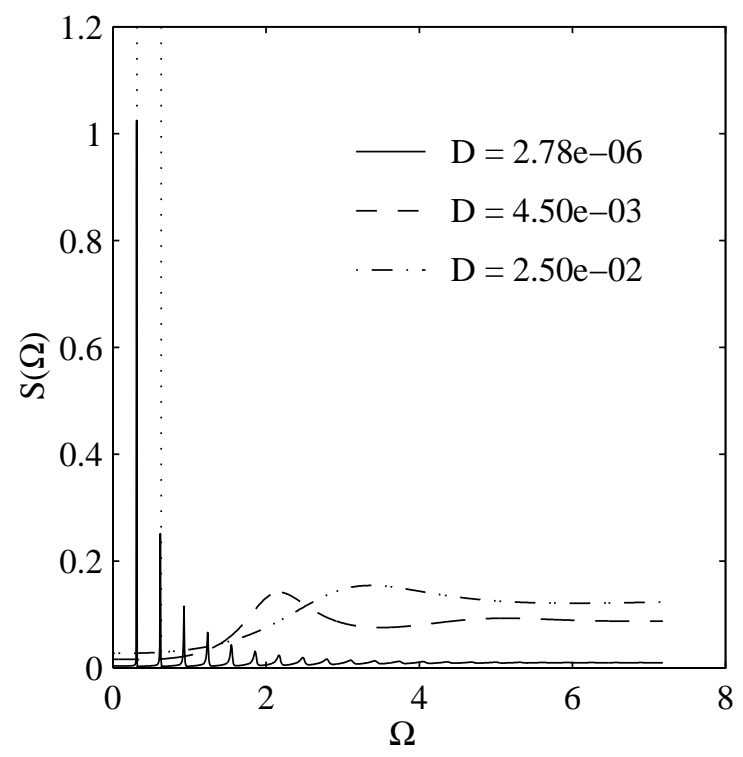

Fig. 1. Power spectral density for $\mu=0.97, q=0.03, \omega=0.1 \pi, \epsilon=0.0014$ for three different noise levels, corresponding to $D_{\max }$, medium and high noise. The vertical dotted lines mark the input frequency $\omega$ and its first harmonic.

In defining the signal-to-noise ratio, the following difficulty arises. The reset mechanism introduces a second timescale into the system besides the one given by the input frequency. Therefore, the output spectrum instead of spikes will have maxima of finite width, and the locations $\Omega_{\mathrm{s}}$ of these are shifted away from the input frequency $\omega$ (Fig. 1). We thus search a neighborhood of the input frequency for the signal peak and define

$$
\begin{aligned}
\mathrm{SNR} & =\frac{\max \{S(\Omega) \mid(1-\alpha) \omega<\Omega<(1+\alpha) \omega\}}{S_{P}} \\
& =\pi\langle\tau\rangle \max \{S(\Omega) \mid(1-\alpha) \omega<\Omega<(1+\alpha) \omega\} .
\end{aligned}
$$

As discussed above, we use the uniform spectral density $S_{P}$ of the Poissonian spike train with firing rate $1 /\langle\tau\rangle$ as noise reference level. Note that no SNR is calculated if the spectrum is monotonous in $[(1-\alpha) \omega,(1+\alpha) \omega]$.

For all data shown, we have calculated the FPTD $\rho(t)$ up to $t=t_{\text {max }}$ such that $\int_{0}^{t_{\max }} \rho(t) \mathrm{d} t \geq 0.99$. Unless stated otherwise below, we employed a stepsize of $h=0.1$ and set the initial phase of the stimulus to $\varphi=0$. Parameter sets for which $\rho(t)$ assumed negative values were discarded unless the latter could clearly be identified as numerical noise. PSDs were calculated at increasing frequency resolutions until results became consistent. The interval width for searching the signal was chosen as $\alpha=0.07$.

As the central result of our work, we show in Figs. 2 and 3 the dependence of the signal-to-noise ratio on the input noise strength for various values of drift term $\mu$, modulation amplitude $q$ and frequency $\omega$, which correspond to distances-from-threshold $0.001<\epsilon<0.01$. All data clearly show stochastic 


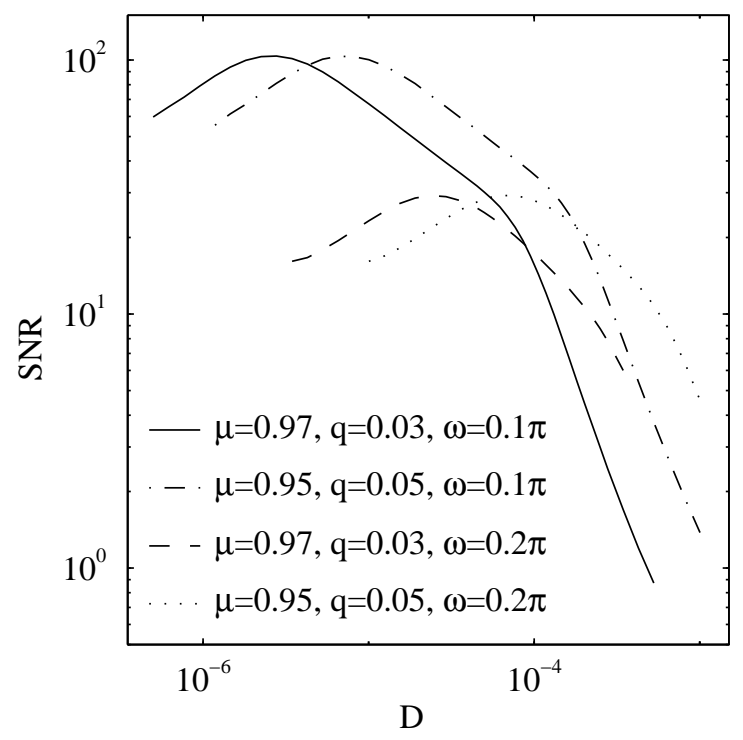

Fig. 2. Signal-to-noise ratio vs. input noise strength for signals with small distance-to-threshold. From top to bottom in the legend: $\epsilon=0.0014,0.0023,0.0046$, 0.0077 .



Fig. 3. Signal-to-noise ratio vs. input noise strength for different input frequencies but the same distance-from-threshold $\epsilon=0.0014$. Here, $\mu=0.95$ and $q=0.05 \times \sqrt{1+\omega^{2}} / \sqrt{1+\omega_{1}^{2}}, \varphi=\cot ^{-1} \omega_{1}-\cot ^{-1} \omega, h=0.1 \times \omega_{1} / \omega, \omega_{1}=0.1 \pi$.

resonance, i.e. attain the maximal signal-to-noise ratio $\mathrm{SNR}_{\max }$ at a noise strength $D_{\max }>0$.

To explain why the SNR peaks, we best turn to the properties of the deterministic solution $\langle x(t)\rangle$ and the FPTD $\rho(t)$, which are shown in Fig. 4 for $\omega=0.1 \pi, \mu=0.97, q=0.03(\epsilon \approx 0.0014)$; this parameter set corresponds to the solid line in Fig. 2 and to the spectra shown in Fig. 1. For strong noise, 


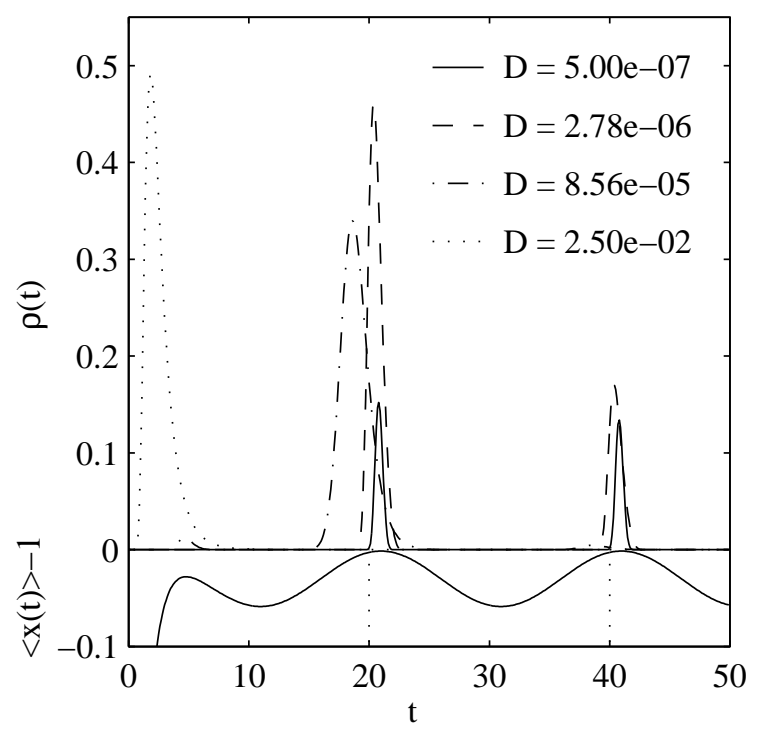

Fig. 4. Deterministic solution and first-passage-time density for $\mu=0.97, q=0.03$, $\omega=0.1 \pi$ and $\epsilon=0.0014$ as in Fig. 1. The noise levels correspond to (from top to bottom): below $D_{\max }$, at $D_{\max }$, at $D_{c}$ and at high noise. The vertical dotted lines mark the first and second period of the input signal. $\langle x(t)\rangle$ has been shifted for clarity.

the modulation of $\langle x(t)\rangle$ becomes virtually negligible and the threshold crossing probability is concentrated in a "drift peak" at small $t$. This drift peak shifts towards $t=0$ and sharpens as the noise strength is increased, becoming similar to a $\Gamma$-distribution (data not shown). In the spectrum, this peak corresponds to a widening hump shifting towards higher frequencies (Fig. 1) and no signal peak is left in the vicinity of the input frequency $\omega$. As the input noise strength $D$ decreases, threshold crossings become concentrated around the maxima of $\langle x(t)\rangle$, and firing events are synchronized to the input stimulus, with the first peak of $\rho(t)$ dominating the distribution for $D_{\max }$. As $D$ is reduced beyond $D_{\max }$, the peak at the first period shrinks and the firing probability is more evenly distributed over subsequent maxima of $\langle x(t)\rangle$. Therefore, a variable number of maxima is skipped before the threshold is reached, resulting in erratic firing and thus a decrease in SNR (Fig. 4).

Obviously, we cannot expect stochastic resonance for $\epsilon \leq 0$ in this system, for if the deterministic solution $\langle x(t)\rangle$ reaches the threshold, spikes will be perfectly synchronized for $D=0$, although the firing frequency may be far from the frequency of the input signal.

For small distances-from-threshold $(\epsilon<0.003)$ and low frequency $(\omega=0.1 \pi)$, we observe stochastic resonance at very small noise strengths $D_{\max }$, and the SNR decays algebraically as $D$ is increased beyond $D_{\max }$ (Fig. 2). Furthermore, this decay exhibits a crossover between two regimes at an intermediate noise strength $D_{c}$. For $D<D_{c}$, the loss in SNR is due to the widening of the peaks 


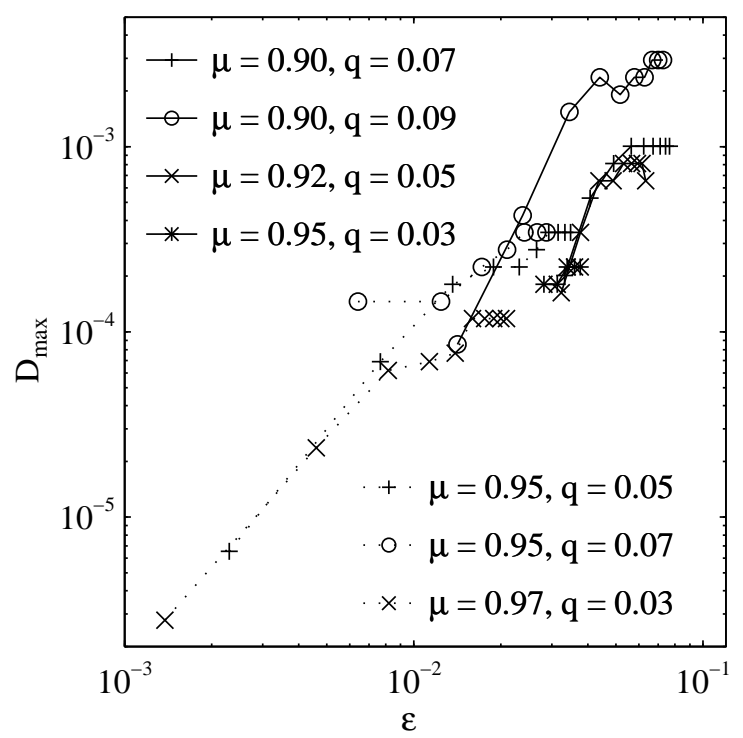

Fig. 5. Position of SNR maximum vs. distance-from-threshold. Data pertaining to identical values of $\mu$ and $q$ but different $\omega$ are connected by lines. Note that $D_{\max }$ was chosen from the set of noise strengths for which calculations were performed, leading to discretization effects along the ordinate.

in the FPTD, which are located at the maxima of $\langle x(t)\rangle$, while for $D>D_{c}$, the drift peak becomes clearly discernible, corresponding to the onset of firing not synchronized with the input stimulus, see Fig. 4.

If $\langle x(t)\rangle$ remains further from thresold, either due to reduced $q$ or increased $\omega$, stochastic resonance occurs at higher input noise strengths $D_{\max }$ and yields smaller maximum values of SNR, see the lower two curves in Fig. 2. This is to be expected, because as the deterministic solution remains smaller, the noise contribution to threshold crossing must increase, reducing the synchronization of firing events with maxima of $\langle x(t)\rangle$.

The input noise strength $D_{\max }$ at which SNR attains its maximum depends strongly on the distance-from-threshold $\epsilon$, as is demonstrated in Fig. 5. Here, we have plotted $D_{\max }$ vs. $\epsilon$ on a double-logarithmic scale. Indeed, the location of the SNR maximum roughly obeys a power law $D_{\max } \sim \epsilon^{\gamma}$. A least squares fit yields $\gamma \approx 1.5$. The detailed dependency of $D_{\max }$ on $\epsilon$ is quite complex, though, and not yet well understood.

On the other hand, $D_{\max }$ hardly depends on the input frequency $\omega$ if the input amplitude $q$ is adjusted so as to obtain the same distance-from-threshold for all frequencies, see Fig. 3. This behavior is to be expected from the mechanism suggested above: the maximal SNR is reached as the firing probability is concentrated at the maxima of $\langle x(t)\rangle$. 


\section{Conclusions and Perspectives}

In this letter, we have investigated the response of a model neuron with reset mechanism to sinusoidal input with additive white noise. The inter-spikeinterval was determined by an efficient numerical method and power spectral densities were obtained by exploiting the renewal properties of the spike train generated. These techniques permitted us to study the behavior of the model neuron over a wide range of parameters, especially at very low noise strengths. We found clear evidence for stochastic resonance, i.e. the signal-to-noise ratio of the neuron's output shows a distinct maximum at non-vanishing input noise. Further, we have proposed a mechanism underlying this effect. The results suggest that nature does indeed employ stochastic resonance to obtain optimal signal-to-noise ratios in an inherently noisy information processing system. A detailed comparison with neurophysiological data will be given elsewhere.

In future work, two questions need to be addressed. The dependence of the neuron's response on the phase $\varphi$ of the input stimulus has yet to be studied in detail. We expect such work to shed more light on the detailed structure of the dependencies of the signal-to-noise ratio on the input noise strength and of the position of the SNR maximum on the distance-from-threshold. More importantly, though, our model shares a weakness with other studies of integrate-and-fire neurons [14,32]: the presumed phase reset of the input stimulus is not very plausible from the viewpoint of neurophysiology. Work on an extended model overcoming this difficulty is currently in progress.

\section{Acknowledgements}

The authors thank M. Katakame for inspiring discussions. H. E. Plesser received partial support from Studienstiftung des deutschen Volkes.

\section{References}

[1] R. Benzi, A. Sutera, and A. Vulpiani. The mechanism of stochastic resonance. J. Phys. A, 14:L453-L457, 1981.

[2] S. Fauve and F. Heslot. Stochastic resonance in a bistable system. Phys. Lett. A, 97:5-7, 1983.

[3] B. McNamara, K. Wiesenfeld, and R. Roy. Observation of stochastic resonance in a ring laser. Phys. Rev. Lett., 60:2626-2629, 1988. 
[4] A. Longtin, A. Bulsara, and F. Moss. Time-interval sequences in bistable systems and the noise-induced transmission of information by sensory neurons. Phys. Rev. Lett., 67:656-659, 1991.

[5] J. E. Levin and J. P. Miller. Broadband neural encoding in the cricket cercal sensory system enhanced by stochastic resonance. Nature, 380:165-168, 1996.

[6] K. Wiesenfeld and F. Moss. Stochastic resonance and the benefits of noise: from ice ages to crayfish and SQUIDs. Nature, 373:33-36, 1995.

[7] B. McNamara and K. Wiesenfeld. Theory of stochastic resonance. Phys. Rev. A, 39:4854-4869, 1989.

[8] P. Jung. Periodically driven stochastic systems. Physics Reports, 234:175-295, 1993.

[9] J. J. Collins, C. C. Chow, and T. T. Imhoff. Aperiodic stochastic resonance in excitable systems. Phys. Rev. E, 52:R3321-R3324, 1995.

[10] T. Zhou, F. Moss, and P. Jung. Escape-time distributions of a periodically modulated bistable system with noise. Phys. Rev. A, 42:3161-3169, 1990.

[11] A. Bulsara, E. W. Jacobs, T. Zhou, F. Moss, and L. Kiss. Stochastic resonance in a single neuron model: theory and analog simulation. J. theor. Biol., 152:531$555,1991$.

[12] H. C. Tuckwell. Stochastic Processes in the Neurosciences. SIAM, Philadelphia, 1989.

[13] P. Lánský and J. P. Rospars. Ornstein-Uhlenbeck model neuron revisited. Biol. Cybern., 72:397-406, 1995.

[14] A. R. Bulsara, T. C. Elston, C. R. Doering, S. B. Lowen, and K. Lindenberg. Cooperative behavior in periodically driven noisy integrate-fire models of neuronal dynamics. Phys. Rev. E, 53:3958-3969, 1996.

[15] K. Wiesenfeld, D. Pierson, E. Pantazelou, and F. Moss. Stochastic resonance on a circle. Phys. Rev. Lett., 72:2125-2129, 1994.

[16] P. Jung. Threshold devices: fractal noise and neural talk. Phys. Rev. E, 50:25132522, 1994.

[17] Z. Gingl, L. B. Kiss, and F. Moss. Non-dynamical stochastic resonance: theory and experiments with white and arbitrarily coloured noise. Europhys. Lett., 29:191-196, 1995.

[18] P. Jung. Stochastic resonance and optimal design of threshold detectors. Phys. Lett. A, 207:93-104, 1995.

[19] P. Lánský, L. Sacerdote, and F. Tomassetti. On the comparison of Feller and Ornstein-Uhlenbeck models of neural activity. Biol. Cybern., 73:457-465, 1995.

[20] J. Inoue, S. Sato, and L. M. Ricciardi. On the parameter estimation for diffusion models of single neuron's activities. Biol. Cybern., 73:209-221, 1995. 
[21] D. R. Cox and P. A. W. Lewis. The Statistical Analysis of Series of Events. Methuen, London, 1966.

[22] N. G. van Kampen. Stochastic Processes in Physics and Chemistry. NorthHolland, Amsterdam, second edition, 1992.

[23] E. Schrödinger. Zur Theorie der Fall- und Steigversuche an Teilchen mit Brownscher Bewegung. Physikalische Zeitschrift, 16:289-295, 1915.

[24] L. M. Ricciardi and S. Sato. First-passage-time density and moments of the Ornstein-Uhlenbeck process. J. Appl. Prob., 25:43-57, 1988.

[25] A. J. F. Siegert. On the first passage time probability problem. Phys. Rev., 81:617-623, 1951.

[26] W. H. Press, S. A. Teukolsky, W. T. Vetterling, and B. P. Flannery. Numerical Recipes in C. Cambridge University Press, Cambridge, GB, second edition, 1992.

[27] A. Buonocore, A. G. Nobile, and L. M. Ricciardi. A new integral equation for the evaluation of first-passage-time probability densities. Adv. Appl. Prob., 19:784-800, 1987.

[28] V. Giorno, A. G. Nobile, L. M. Ricciardi, and S. Sato. On the evaluation of first-passage-time probability densities via non-singular integral equations. Adv. Appl. Prob., 21:20-36, 1989.

[29] M. S. Bartlett. The spectral analysis of point processes. J. R. Statist. Soc. Ser. B, 29:264-296, 1963.

[30] D. J. Daley and D. Vere-Jones. An Introduction to the Theory of Point Processes. Springer, New York, 1988.

[31] D. H. Perkel, G. L. Gerstein, and G. P. Moore. Neuronal spike trains and stochastic point processes. Biophys J, 7:391-418, 1967.

[32] A. R. Bulsara, S. B. Lowen, and C. D. Rees. Cooperative behavior in the periodically modulated Wiener process: noise-induced complexity in a model neutron [sic]. Phys. Rev. E, 49:4989-5000, 1994. 